\title{
Relevance of an extensive follow-up after surgery for nonsmall cell lung cancer
}

\author{
Delphine Gourcerol ${ }^{1,2}$, Arnaud Scherpereel ${ }^{1,2}$, Stephane Debeugny $^{3}$, \\ Henri Porte ${ }^{2,4}$, Alexis B. Cortot ${ }^{1,2}$ and Jean-Jacques Lafitte ${ }^{1,2}$ \\ Affiliations: \\ 'Pulmonary and Thoracic Oncology Dept, Lille University Hospital (CHRU), Lille, \\ ${ }^{2}$ University of Lille Nord de France, Lille, \\ ${ }^{3}$ Medical Informatic Dept of Pau Hospital, Pau, and \\ ${ }^{4}$ Thoracic Surgery Dept, Lille University Hospital (CHRU), Lille, France.
}

\section{Correspondence:}

D. Gourcerol, Service de Pneumologie, Boulevard Hauterive, Centre Hospitalier François Mitterrand, (F)64000 Pau, France.

E-mail: delgourceroldgmail.com

ABSTRACT There are no international guidelines for an appropriate and cost-effective follow-up for resected nonsmall cell lung cancer (NSCLC).

We retrospectively reviewed the outcome of NSCLC patients after curative surgery. Follow-up included physical examination and chest radiography every 3 months, and chest computed tomography (CT) scan, bronchoscopy, abdominal ultrasound, brain CT scan and bone scan every 6 months for 3 years, then every year over the following 2 years. Prognostic factors and costs were analysed.

Median overall survival following surgery for NSCLC in 162 patients was 38.5 months. Recurrence occurred in 85 (52.5\%) patients including 41 (48\%) symptomatic subjects. Disease-free survival was similar between patients with asymptomatic recurrence versus symptomatic patients (11.4 versus 12 months; $\mathrm{p}=0.41)$. Recurrence was detected by physical examination or chest radiography in $47(55.3 \%)$ patients. Curative-intent therapy was provided in $18(41 \%)$ out of 44 patients with asymptomatic recurrence and in four $(10 \%)$ out of 41 symptomatic cases $(\mathrm{p}=0.001)$. Median overall survival from time of recurrence was higher in asymptomatic patients than in symptomatic patients ( 15.5 versus 7.2 months; $p=0.001)$. The cost per year of life gained was USD32 700 (€22 397).

An extensive follow-up, with acceptable cost, may improve the outcome of patients with resected NSCLC through detection of asymptomatic recurrences; however, validation by prospective studies is required.

@ERSpublications

An extensive follow-up, with acceptable cost, may improve the outcome of patients with resected NSCLC http://ow.ly/nUOez

For editorial comments see page 1178.

Received: June 012012 | Accepted after revision: Jan 292013 | First published online: March 212013

Conflict of interest: Disclosures can be found alongside the online version of this article at www.erj.ersjournals.com 


\section{Introduction}

In current guidelines, surgery is the most appropriate treatment for early stage nonsmall cell lung cancer (NSCLC). However, only $50-60 \%$ of these patients will survive $>5$ years after surgery. A recurrence may occur in about $50 \%$ of patients [1]. There are no internationally validated guidelines for an appropriate and cost-effective follow-up after surgery for NSCLC. Wide variations in follow-up modalities are observed in Europe and in the USA $[2,3]$. On the basis of retrospective studies [4-7], it is usually considered that there is no rationale for procedures other than physical examinations and chest radiography in patients with resected lung cancer. Only one prospective study [8] has evaluated the feasibility and impact of a standardised intensive post-surgery follow-up; it showed a significant survival advantage in patients with asymptomatic relapses, compared to symptomatic patients. To confirm the potential value of an extensive follow-up in resected NSCLC, a randomised trial should be undertaken, as was carried out in breast cancer and colorectal cancer patients $[9,10]$. However, it still remains unclear whether follow-up in these patients is cost-effective and whether it can improve the outcome due to an early detection of relapse.

We performed a retrospective study, including a cost-effectiveness analysis, to assess the feasibility and the impact of a standardised intensive follow-up on outcome of NSCLC patients following curative surgery in our institution.

\section{Patients and methods}

Patients

The records of all patients, from the Pulmonary and Thoracic Oncology Department of the Lille University Hospital, Lille, France, who underwent resection for NSCLC between January 1990 and December 2002, were retrospectively reviewed. Inclusion criteria included a definitive diagnosis of NSCLC, a complete follow-up performed in our department for at least 5 years after surgery, no previous malignancy in the 5 years before NSCLC surgery and a complete surgical resection. Patients having complete resection of synchronous brain metastasis before NSCLC surgery were eligible. Patients who died within 30 days after surgery were excluded from the study. The design of the study was approved by the local ethics committee of our institution.

\section{Follow-up procedures}

During the first 3 years after surgery, follow-up included the following procedures: physical examination and chest radiography every 3 months; chest computed tomography (CT) scan, fibreoptic bronchoscopy, abdominal ultrasound, brain CT scan, and bone scan every 6 months.

During the next 2 years, physical examination and chest radiography were performed every 3 months, and chest CT scan, fibreoptic bronchoscopy, abdominal ultrasound, brain CT scan, and bone scan were performed once a year.

\section{Compliance with follow-up procedures}

The number of chest radiographs, chest CT scans, fibreoptic bronchoscopies, abdominal ultrasounds, brain CT scans and bone scans performed until recurrence, death or last date of follow-up in patients without disease recurrence was calculated and compared with the number of scheduled procedures. The frequency of physical examinations was the same as the frequency of chest radiography, as they were performed at the same time.

\section{Recurrences}

The criteria reported by MARTINI et al. [11] were used to differentiate second primary lung cancers and recurrences of lung cancer. Patients having tumour recurrence were recorded as being either symptomatic (symptoms or abnormal physical examination) or asymptomatic at the time of diagnosis of the recurrence. The method leading to the diagnosis of recurrence was reported. Treatment of recurrence was considered as curative if the patient had complete surgical resection or irradiation for a local recurrence or a second primary lung cancer, or if they had complete surgical resection of a brain solitary metastasis with a controlled local disease. Chemotherapy for advanced disease, brain radiotherapy and palliative surgery were considered as palliative treatments.

\section{Cost analysis}

The cost analysis was performed as described by WEESTEL et al. [8]. The cost per years of life potentially gained by the extra procedures (chest CT scan, fibreoptic bronchoscopy, abdominal ultrasound, brain CT scan and bone scan) was estimated by dividing the cost of "extra investigation" during the median diseasefree survival duration for the whole population (A) by the numbers of years gained (B), i.e. cost per years of life gained=A/B. 
128.4

56.7

96

193

128.4

The cost of extra procedures (A) was calculated per patient for the median disease-free survival duration for the whole population (the studied period). Reimbursement prices are determined for each procedure by the French healthcare system. The French healthcare system repayment rates and money exchange rates ( $€ 1$ is equal to \$1.46) for 2007 were used (table 1). For one patient, the cost of the follow-up programme was the total of the costs for each procedure during the studied period, multiplied by the number of times (a) this procedure was supposed to be performed during the studied period.

$$
\begin{aligned}
\mathrm{A}= & (\mathrm{a} \times \text { chest CT scan }+\mathrm{a} \times \text { fiberoptic bronchoscopy }+\mathrm{a} \times \text { abdominal ultrasound }+ \\
& \mathrm{a} \times \text { brain CT scan }+\mathrm{a} \times \text { bone scan }) \times \mathrm{n}
\end{aligned}
$$

Where $\mathrm{n}$ is the number of patients.

The number of years of life gained by "extra investigations" (B) was the number of asymptomatic patients alive 3 years after recurrence $(\mathrm{X})$ multiplied by three, among asymptomatic patients who had a recurrence detected by the "extra investigations" and treated with curative intent, as the benefit of an intensive follow-up is mostly for the patients whose recurrences would not have been found in a clinical follow-up, i.e. $\mathrm{B}=3 \mathrm{X}$.

\section{Survival and statistical analysis}

The date of first treatment (surgery or neo-adjuvant chemotherapy) was considered as day one. The time from first treatment to death or last observation was defined as the overall survival. The disease-free interval was defined as the time from first treatment to the detection of recurrence (or date of death or last observation in patients without recurrence). Survival from time of recurrence was also analysed. Survival curves were calculated using the Kaplan-Meier method. Survival differences between groups were evaluated by the log-rank test. Factors analysed for prognostic significance included sex, age at first treatment, histology (squamous cell carcinoma versus other types), disease-free survival from surgery, mode of presentation (asymptomatic versus symptomatic patients), diagnostic procedure (physical examination or chest radiography versus extra-procedures), site of recurrence (thorax only versus extra-thoracic or both thoracic and extra-thoracic). Multivariate analysis of variables with statistical significance was performed using Cox regression. For the survival analyses, hazard ratio (HR) and the corresponding 95\% confidence intervals (CIs) were assessed. Statistically significant differences are indicated by $\mathrm{p}<0.05$. All statistical analyses were performed using SAS 9.1.3 (SAS Institute Inc., Cary, NC, USA).

\section{Results}

\section{Patients' characteristics}

Between January 1990 and December 2007, 197 patients were followed, in the Pulmonary and Thoracic Oncology Department of the Lille University Hospital, after curative intent resection of a NSCLC. 35 patients were excluded from the study due to incomplete resection $(n=4)$, previous malignancy in the 5 years before surgery $(n=14)$ or death within the first 30 days following surgery $(n=17)$. The remaining 162 patients were included in this study.

Baseline characteristics of the patients included in the study are detailed in table 2. The median (range) age was 59 years (31-81 years). Most of the patients were male $(90.7 \%)$ and had squamous cell carcinoma (57\%). Two patients had stage IV disease and had resection of both the primary tumour and a solitary brain metastasis. The median (range) follow-up period was 38.2 months (1.5-213 months).

\section{Compliance with follow-up procedures}

Most of the procedures were correctly performed in patients at the right time. In fact, $86 \%$ of the scheduled physical examinations and chest radiography were performed; $76.5 \%$ of chest CT scans, $68 \%$ of bronchoscopies, $67.5 \%$ of abdominal ultrasounds, $52.5 \%$ of bone scans and $24 \%$ of brain CT scans were also carried out as planned. 
TABLE 2 Characteristics of the 162 patients included in the study

\section{Characteristics}

$\begin{array}{lc}\text { Age years } & 59(31-81) \\ \text { Sex } & 147(90.7) \\ \text { Male } & 15(9.3) \\ \text { Female } & \\ \text { Histologic subtypes of NSCLC } & 92(57) \\ \text { Squamous cell carcinoma } & 61(37.5) \\ \text { Adenocarcinoma } & 6(3.5) \\ \text { Large cell carcinoma } & 3(2) \\ \text { NSCLC not otherwise specified } & 23(14.2) / 56(34.6) \\ \text { Stage (1997 TNM System) } & 4(2.5) / 27(16.7) \\ \text { la/lb } & 37(22.8) / 13(8) \\ \text { Ila/IIb } & 2(1.2) \\ \text { IIla/IIlb } & \\ \text { IV } & \\ \text { Resection } & 36(22.2) \\ \text { Pneumonectomy } & 23(14.2) \\ \quad \text { Total } & 13(8) \\ \text { Left } & 106(65.4) / 13(8) \\ \text { Right } & 7(4.4) \\ \text { Lobectomy/bilobectomy } & \\ \text { Atypic resection } & 89(55) \\ \text { Perioperative therapy } & 73(45) \\ \text { Surgery only } & 5(3.1) \\ \text { Surgery and perioperative therapy } & 4(2.5) \\ \text { Induction chemotherapy } & 45(27.7) \\ \text { Consolidation chemotherapy } & 19(11.7) \\ \text { Consolidation radiotherapy } & \end{array}$

Data are presented as median (range) or n (\%). NSCLC: nonsmall cell lung cancer; TMN: tumour, node, metastasis.

\section{Recurrences}

A recurrence occurred in 85 (52.5\%) patients and was symptomatic in 41 (48\%) patients (fig. 1). 40 (47\%) patients had thoracic recurrence, $26(30.5 \%)$ patients had extra-thoracic recurrence and 19 (22.5\%) patients had both thoracic and extra-thoracic recurrence.

Significantly more patients with thoracic recurrence were asymptomatic than were symptomatic: 27 $(67.5 \%)$ versus $13(32.5 \%)$ patients, respectively $(\mathrm{p}=0.006)$. In contrast, significantly more of the patients with extra-thoracic \pm thoracic recurrence(s) were symptomatic than were asymptomatic: 28 (62\%) versus 17 $(38 \%)$ patients. The sites of thoracic recurrence were: the pleura $(n=1)$, pulmonary metastases $(n=6)$, mediastinum $(n=14)$, a second primary cancer $(n=13)$, pulmonary lymphangitis $(n=1)$, the thoracotomy scar $(n=1)$, and a combination of several thoracic sites $(n=4)$.

Recurrence was detected by physical examination or chest radiography in 47 (55.3\%) patients, and by another procedure in $38(44.7 \%)$ patients. Among the 47 patients whose recurrence was detected by physical examination or chest radiography, 61 sites of recurrence were detected as some patients may recur in several sites Among the patients whose recurrence was detected by another procedure, recurrence was diagnosed by chest CT scan in $32(52.5 \%)$ patients, bronchoscopy in $12(19.5 \%)$ patients, abdominal ultrasound in six $(10 \%)$ patients, bone scan in four $(6.5 \%)$ patients, and brain CT scan in seven (11.5\%) patients. As some patients could have several recurrences, several procedures could be positive for the same patient.

Following recurrence, $22(26 \%)$ out of 85 patients were treated with curative intent including surgery in 11 $(50 \%)$ patients, with five patients undergoing thoracic surgery and six patients undergoing resection of a single metastasis (four brain metastases, one adrenal metastasis and one supra-clavicular lymph node metastasis); radiotherapy in $10(45 \%)$ patients with thoracic irradiation in seven patients (two subjects also had brachytherapy), thoracic irradiation and brachytherapy in two patients and brachytherapy alone in one patient; and local treatment with endobronchial laser therapy in one (5\%) patient.

$44(52 \%)$ patients were treated with palliative intent (chemotherapy and radiotherapy). 19 (22\%) patients received best supportive care alone. 


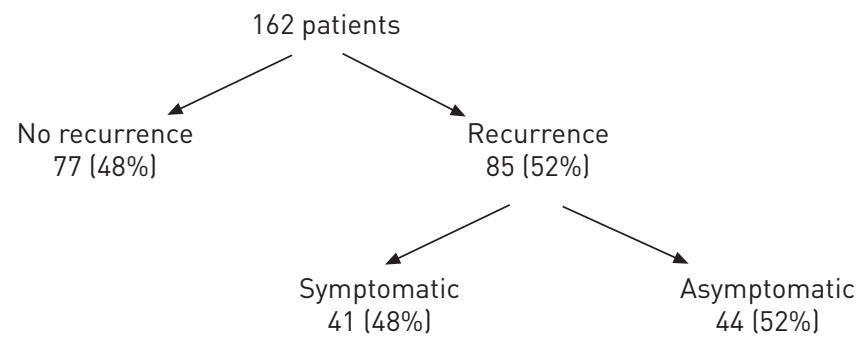

Site of recurrence

\section{Diagnostic procedure}

Clinical examination or chest radiography

Other investigation
0
6
$47(55.3 \%)$

$38(44.7 \%)$

FIGURE 1 Symptoms, sites and diagnostic procedures at the time of recurrence.

Treatment was delivered with curative intent in $18(41 \%)$ out of the 44 asymptomatic patients and in four $(10 \%)$ of the 41 symptomatic patients $(p=0.001) .20(45 \%)$ asymptomatic patients and $24(58.5 \%)$ symptomatic patients received palliative therapies $(\mathrm{p}=0.003)$. Best supportive care alone was proposed for six $(13.5 \%)$ asymptomatic patients and $13(32 \%)$ symptomatic patients $(\mathrm{p}=0.003)$.

Treatment was delivered with curative intent in 15 (37.5\%) of the 40 patients who had a thoracic recurrence only and in seven $(15 \%)$ of the 45 patients who had an extra-thoracic recurrence or both thoracic and extrathoracic recurrences $(\mathrm{p}=0.021)$.

\section{Survival}

Median overall survival following initial surgery was 38.5 months. No significant difference in the diseasefree period was found between asymptomatic patients and symptomatic patients (11.4 versus 12 months, $\mathrm{p}=0.41, \mathrm{HR}=1.20,95 \%$ CI $0.78-1.86$ ). Median survival from the time of recurrence was significantly higher in patients with asymptomatic recurrence than in patients with symptomatic recurrence (15.5 versus 7.2 months, $\mathrm{p}=0.001, \mathrm{HR}=2.09,95 \%$ CI 1.33-3.28) (fig. 2).

The results of univariate analysis of survival are detailed in table 3. The absence of symptoms at the time of recurrence, the diagnostic procedure (physical examination and chest radiography versus other procedures)

FIGURE 2 Survival of resected nonsmall cell lung cancer patients from the time of their recurrence, according to whether or not symptoms were present at the time of recurrence. Open circles indicate censored points. HR: hazard ratio.

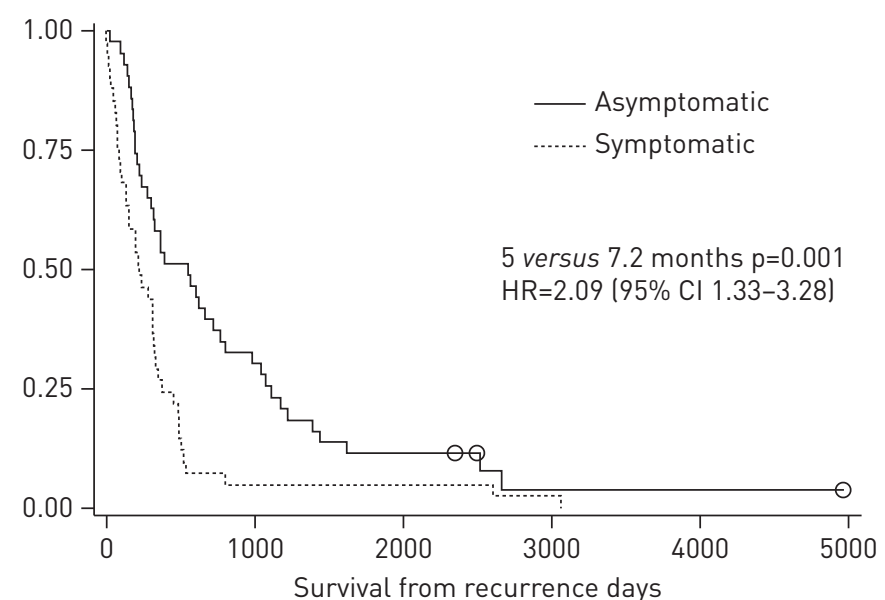


TABLE 3 Univariate and multivariate analysis of prognostic factors for survival from the time of recurrence in patients with resected nonsmall cell lung cancer

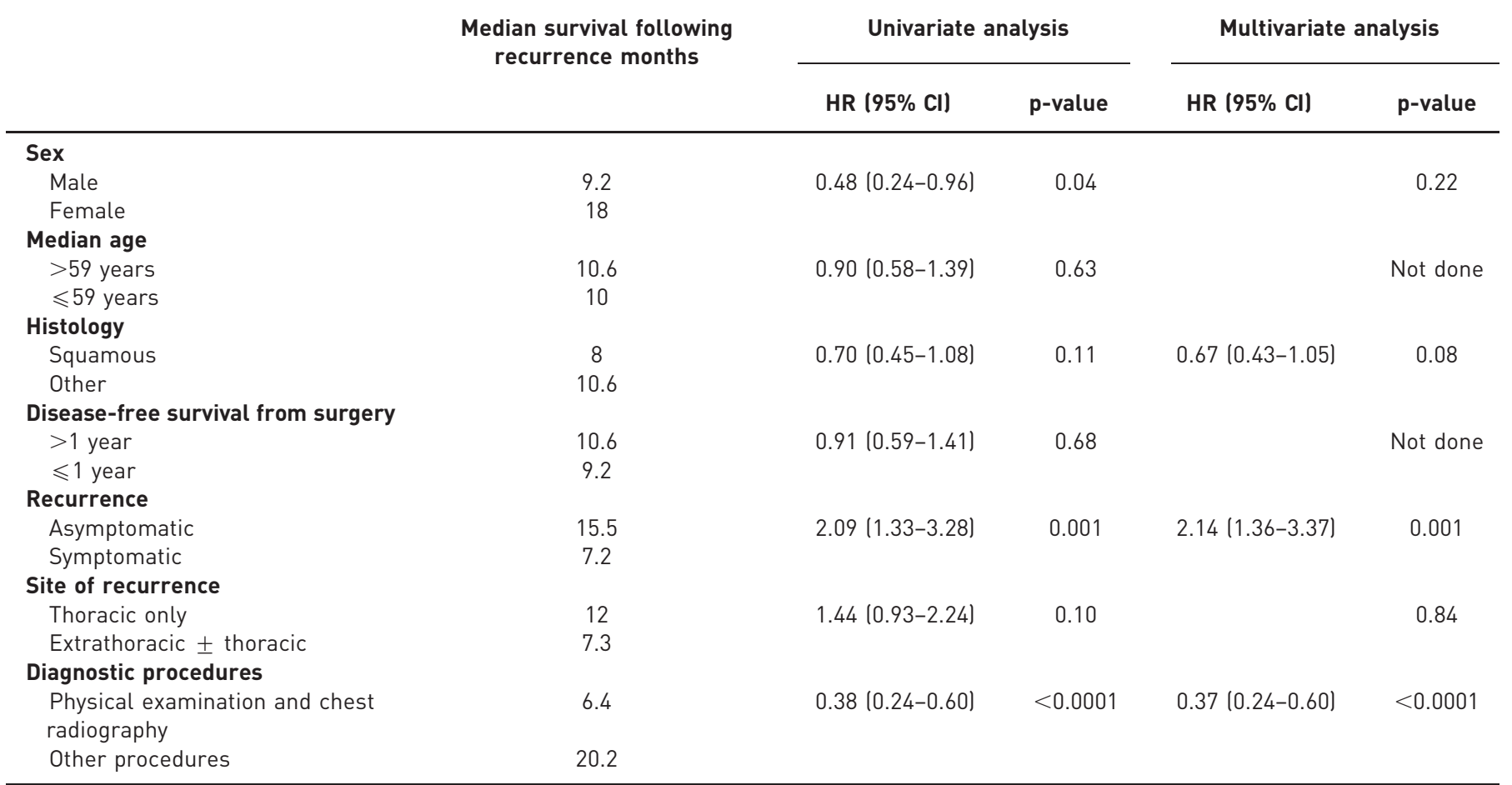

used to diagnose the recurrence and sex were found to be significantly associated with a better survival from the time of recurrence. Age, site of the recurrence, histology and disease-free period did not significantly affect the outcome.

In multivariate analysis, the diagnostic procedure for the diagnosis of recurrence and the absence of symptoms at the time of recurrence were the most significant factors for predicting survival after recurrence (table 3 ).

\section{Cost analysis}

For a follow-up period of 32 months (equal to the median disease-free survival), the cost per patient was USD6660 (€4561). 11 patients having an asymptomatic recurrence detected by a procedure other than physical examination or chest radiography were treated with curative intent and were still alive 3 years after recurrence. Therefore, the number of gained years of life was 33. The total cost incurred by procedures other than physical examination or chest radiography (chest CT scan, bronchoscopy, abdominal ultrasound, bone scan and brain CT scan) was USD1 078920 ( $€ 1078920)$. Thus the cost per year of life gained was USD32 700 (€22 397).

\section{Discussion}

In this study, we found that an intensive follow-up of patients with resected NSCLC is feasible, cost-effective and may improve survival by detecting asymptomatic recurrence. Despite the high number of planned procedures in the follow-up programme, overall compliance was good ( $76 \%$ for chest CT scan). However, it was impossible to assess the impact of some examinations, such as the brain CT scan, in the follow-up of these patients as the compliance for this procedure was very low (24\%). A major limitation in our study is the lack of a control group, as it is retrospective and we followed up all of our patients with an intensive programme. The second limitation is linked to the long duration of the study: we already performed neoadjuvant and adjuvant therapy as it is currently recommended, but the quality of imaging procedures has changed, especially in the case of CT scans. Thus, abdominal ultrasound is not actually the modality recommended for diagnosis of hepatic recurrences because it is less sensitive than a CT scan. Another limitation of the study is that our patients recruited before 2007 were still logically assessed according to the 1997 TNM (tumour, node, metastasis) staging system. Due to the limitations of a retrospective study, it was not feasible to reassess our patients' TNM stage according to the most recent (7th) edition of the lung cancer TNM staging system [12], without the risk of approximating or even providing false data, in 
particular for the " $\mathrm{T}$ " parameter. Therefore, we decided to keep the well characterised TNM stage data from the earlier 1997 TNM classification. Finally, positron emission tomography (PET) scans were not available at this time in the north of France for the follow-up of most of these patients, and, thus, could not be assessed in this study.

The rate of asymptomatic recurrences (52\%) in our study was higher than previously reported (usually 25-30\%), which could be due to a more extensive follow-up programme $[5,8]$. Importantly, we found that asymptomatic patients at the time of recurrence had a significantly longer survival from the time of recurrence than symptomatic patients. Lead time bias cannot explain this survival advantage since no difference in disease-free survival was found between asymptomatic and symptomatic patients. We found that asymptomatic patients at the time of recurrence were more likely to receive any treatment than symptomatic patients, especially curative intent therapy $(41 \%$ versus $10 \%, \mathrm{p}=0.001)$.

WEESTEL et al. [8] previously showed that among patients benefiting from an intensive follow-up programme, including bronchoscopy and chest CT scan with sections of the liver and adrenal glands, asymptomatic subjects at the time of recurrence had a better survival from the time of recurrence than symptomatic patients. In another study, WALSH et al. [5] retrospectively reviewed the records of 358 resected lung cancer patients. Despite discrepancies in the follow-up plan, they also found that asymptomatic patients at the time of recurrence had a significantly longer survival than symptomatic patients. Thus, patients with resected NSCLC may benefit from a follow-up strategy that allows the detection of more asymptomatic recurrences than periodic physical examinations and chest radiography would do.

A better prognosis for patients with asymptomatic recurrences could also be explained because they may be treated with curative intent, for example in case of rare single (brain or adrenal) metastasis or of unique thoracic recurrence (mediastinal lymph nodes, lung metastasis or second lung cancer). In fact, most symptomatic patients did not receive curative intent treatment. These symptomatic patients mainly had extra-thoracic recurrences that were usually not accessible to curative intent treatment at this time, and they had poorer performance status than patients with intrathoracic recurrences. However, we were not able to retrospectively collect reliable performance status data, at the time of recurrence from all patients, allowing us to fully analyse this parameter. Thus, this represents a limitation of our study. Accordingly, patients with thoracic recurrences were usually asymptomatic, except at a late stage, and could not benefit from a curative intent treatment.

Follow-up guidelines for NSCLC patients following resection should take into account benefits in terms of patient outcome, but also quality of life and cost-effectiveness. Although an intensive follow-up might improve quality of life by a better diagnosis, the treatment of potential tobacco-related comorbidities and of post-operative symptoms, it might also be deleterious because of the potential anxiety generated by repeated procedures. Physicians should also take into account the radiation exposure risks related to repeated scanning, even at the low doses similar to CT-scan procedure proposed for lung cancer screening $[13,14]$. There is no published study evaluating follow-up strategies after lung cancer resection that includes a quality-of-life questionnaire. In breast cancer, the GIVIO study analysed the psychological impact of the follow-up [9]. The authors concluded that the patients wanted a strict follow-up; there was no impact on their quality of life because the follow-up did not reassure them but was not stressful either.

The cost of the intensive follow-up we used (USD32 700 or €22397) can be considered acceptable, considering that in the USA, USD50000 is regarded as the upper limit of acceptable cost-effectiveness $[7,15]$. Without the bone scan, which does not diagnose curable recurrences, this cost would even be decreased to USD25 800. Our results are consistent with those from WeESTEL et al. [8] who also found an acceptable cost per gained year of life (USD13 000) in the follow-up of resected NSCLC. The lower cost per gained year of life observed in their study may be due to a less extensive follow-up, and a shorter median disease-free survival (19 months) than in our study (32 months). As our study is more recent than that of WEESTEL et al. [8], a more accurate staging before surgery, due to technological improvements, may explain the low recurrence rate in our study. Younes et al. [6] retrospectively compared the cost-effectiveness of two different follow-up plans after NSCLC resection in 130 patients: a strict one (frequent visits, chest radiography, chest CT scan and liver function test) and another one based on symptoms [16]. The costs of follow-up for the strict plan group were significantly higher than those for the symptoms plan group, with no difference in survival after recurrence between the two groups. However, the follow-up only lasted 2 years after resection and recurrences occurred in 32 (24\%) patients, 14 patients in the strict group and 18 patients in the symptom group, limiting the statistical analysis of this follow-up role on patients' outcome. In colon cancer, an intensive post-operative follow-up is recommended, and the cost per year of life gained is less than USD10 000 to be compared with a cost per gained year of life of USD64 000 for haemodialysis [4]. KENT et al. [16] created a decision analysis model in which a hypothetical cohort of patients underwent 
annual chest CT after resection of a stage Ia NSCLC. The incidence of a second primary lung cancer, sensitivity and specificity of chest CT scan, as well as survival after resection of the initial primary and second lung cancer were derived from published literature. It showed a cost of surveillance by chest CT scan once a year over 5 years of USD47 676 per quality-adjusted life-year gained [16]. However, several factors could explain a cost-ineffective follow-up by chest CT scan, in this case: 1) an age $>65$ years when starting the follow-up; 2) a cost of chest CT scan higher than USD700; 3) an incidence of second pulmonary lung cancer lower than $1.6 \%$ per patient per year of follow-up; and 4) a false positive rate by chest CT-scan higher than $14 \%$.

In the present study, a survival advantage was shown for asymptomatic patients whose recurrence was diagnosed during an intensive follow-up. Our follow-up programme could still be effective although less extensive and thus cheaper without the bone scan, and if the CT scans were performed every 6 months during the first 2 years instead of the first 3 years of follow-up. We are currently testing this hypothesis in our institution.

An ongoing French prospective randomised trial with cost analysis (IFCT-0302 trial, NCT00198341) aims to compare a 5 year nonintensive follow-up with an intensive follow-up (two-armed study) in patients with resected NSCLC. The nonintensive follow-up includes a physical examination and chest radiography every 6 months during the first 2 years then every year over 3 years. The intensive follow-up includes a physical examination, a chest radiograph as well as a chest CT scan with sections of the liver and adrenal glands, and a bronchoscopy (depending on the histology of the NSCLC) every 6 months during the first 2 years then every year over 3 years. The primary end-point of the study is overall survival. This trial will help determine the appropriate follow-up for patients with resected NSCLC. However, the value of fluorodeoxyglucosePET scans and brain CT scans, and surveillance following new treatments such as stereotactic radiosurgery will still need to be assessed in future trials.

In conclusion, our data suggest that asymptomatic recurrence following curative surgery for NSCLC is associated with a better survival than symptomatic recurrence. An intensive follow-up may improve the outcome for patients with resected NSCLC through detection of recurrence at an asymptomatic stage. The results of ongoing randomised trials are urgently awaited to suggest guidelines for the follow-up of these patients.

\section{References}

1 Vallières E, Sheperd FA, Crowley J, et al. The IASLC Lung Cancer Staging Project: proposals regarding the relevance of TNM in the pathologic staging of small cell lung cancer in the forthcoming (seventh) edition of the TNM classification for lung cancer. J Thorac Oncol 2009; 4: 1049-1059.

2 Johnson FE, Naunheim KS, Coplin MA, et al. Geographic variation in the conduct of patient surveillance after lung cancer surgery. J Clin Oncol 1996; 14: 2940-2949.

3 Nauheim KS, Virgo KS, Coplin MA, et al. Clinical surveillance testing after lung cancer operations. Ann Thorac Surg 1995; 60: 1612-1616.

4 Virgo KS, Naunheim KS, McKirgan LW, et al. Cost of patient follow-up after potentially curative lung cancer treatment. J Thorac Cardiovasc Surg 1996; 112: 356-363.

5 Walsh GL, O'Connor M, Willis KM, et al. Is follow-up of lung cancer patients after resection medically indicated and cost-effective? Ann Thorac Surg 1995; 60: 1563-1570.

6 Younes RN, Gross JL, Deheinzelin D. Follow-up in lung cancer: how often and for what purpose? Chest 1999; 115: 1494-1449.

7 Egermann U, Jaeggi K, Habicht JM, et al. Regular follow-up after curative resection of nonsmall cell lung cancer: a real benefit for patients? Eur Respir J 2002; 19: 464-468.

8 Weestel V, Choma D, Clément F, et al. Relevance of an intensive postoperative follow-up after surgery for nonsmall cell lung cancer. Ann Thorac Surg 2000; 70: 1185-1190.

9 Impact of follow-up testing on survival and health-related quality of life in breast cancer patients. A multicenter randomized controlled trial. The GIVIO Investigators. JAMA 1994; 271: 1587-1592.

10 Desch CE, Benson AB 3rd, Somerfield MR. Colorectal cancer surveillance: 2005 update of an American Society of Clinical Oncology practice guideline. J Clin Oncol 2005; 23: 8512-8519.

11 Martini N, Bains MS, Burt ME, et al. Incidence of local recurrence and second primary tumors in resected stage I lung cancer. J Thorac Cardiovasc Surg 1995; 109: 120-129.

12 Goldstraw P, Crowley J, Chansky K, et al. The IASLC Lung Cancer Staging Project: proposals for the revision of the TNM stage groupings in the forthcoming (seventh) edition of the TNM classification of malignant tumours. J Thorac Oncol 2007; 2: 706-714.

13 The National Lung Screening Trial Research Team, Aberle DR, Adams AM, et al. Reduced lung-cancer mortality with low-dose computed tomographic screening. N Engl J Med 2011; 365: 395-409.

14 Couraud S, Cortot AB, Greillier L, et al. From randomized trials to the clinic: is it time to implement individual lung-cancer screening in clinical practice? A multidisciplinary statement from French experts on behalf of the French intergroup (IFCT) and the groupe d'Oncologie de langue francaise (GOLF). Ann Oncol 2013; 24: 586-597.

15 Schrag D. Defining optimal treatment for stage II colon cancer: does decision analysis help? Gastroenterology 1999; 117: 1005-1008.

16 Kent MS, Korn P, Port JL, et al. Cost effectiveness of chest computed tomography after lung cancer resection: a decision analysis model. Ann Thorac Surg 2005; 80: 1215-1222. 